\title{
Structure and Mechanical Properties of Poly $(\gamma$-methyl glutamate) Films. II.
}

\author{
Junji Watanabe, Michiro Naka, Junichiro Watanabe, \\ Koji WatanABE, and Ichitaro UEMATSU \\ Department of Polymer Chemistry, Tokyo Institute of Technology, \\ 12-1, Ookayama, Meguro-ku, Tokyo 152, Japan.
}

(Received November 2, 1977)

\begin{abstract}
A series of poly $(\gamma$-methyl D-glutamate) (PMDG) films with the different structures were systematically prepared from dichloromethane (DCM) $-N, N$-dimethylformamide (DMF) solutions. The structures of these films were classified into three types, vix., phase $\mathrm{I}$, phase $\mathrm{M}$, and phase $\mathrm{C}$ as obtained in a previous work from DMF, 1, 2-dichloroethane and DCM, respectively. Observation of the PMDG films cast from chloroform-DMF solutions, showed that phase $\mathrm{C}$ arises from the phase which consists of the cholesteric liquid crystal structure, and it was assumed that the crystallites in phase $\mathrm{C}$ should be divided into mosaic blocks. Three mechanical dispersions were observed at $160,190^{\circ} \mathrm{C}$, and above $200^{\circ} \mathrm{C}$. The $\alpha_{1}$ dispersion (at $160^{\circ} \mathrm{C}$ ) was associated with the molecular relaxation in phase $\mathrm{I}$, and further, the $\alpha_{3}$ dispersion (above $200^{\circ} \mathrm{C}$ ) was related to the molecular motion of $\alpha$-helices in phase $\mathrm{M}$ with the cholesteric twisted structure. For the $\alpha_{2}$ dispersion (at $190^{\circ} \mathrm{C}$ ) commonly observed for phase $\mathrm{C}$, two relaxation mechanisms are discussed: the molecular motion of $\alpha$ helices in phase $\mathrm{C}$ and the mutual displacement of the neighboring crystallites. The PMDG films cast from $m$-cresol at various temperatures and the PMDG-poly $(\gamma$-methyl L-glutamate) (PMLG) blend films cast from chloroform solutions, showed a remarkable shift of $\alpha_{2}$ loss peak temperature. To explain this shift in peak temperature, the latter mechanism seems reasonable for the $\alpha_{2}$ dispersion.

KEY WORDS Poly $(\gamma$-methyl glutamate) / Casting History / Cast Film / Mechanical Dispersion / X-Ray Diffraction /
\end{abstract}

Depending on the solvent and polypeptide concentration, a polypeptide molecule may exist in a number of conformations in solution. ${ }^{1}$ Solvents are categorized into random coil and helical types. The random coil solvents are generally very polar and form strong hydrogen bonds with the polypeptide molecules. Dichloroacetic acid (DCA) and trifluoroacetic acid (TFA) are random coil solvents for many polypeptides in dilute solution. Most chlorinated hydrocarbons such as chloroform and DCM, with DMF and $m$-cresol, favor formation of the helical form of the polypeptide.

Poly ( $\gamma$-methyl glutamate) is known to be soluble also in the coil form in such solvents as DCA and TFA, and in the helical form in helicogenic solvents such as $m$-cresol and chloroform. However, when films are prepared from random coil solvents, the chain conformation in the films is mainly in the helical form. On the other hand, the $\beta$ - chain structure has been observed in the films swollen in formic acid and in crystals precipitated from dilute solution containing formic acid. ${ }^{2}$ This structure is also obtained from TFA or DCA solution, depending on the casting velocity. ${ }^{3}$ Thus, when the films are cast from random coil solvents, the chain conformation in the films is not only in the helical form, but also partially in the $\beta$-chain form.

No conformational change is observed when the films are cast from the helicogenic solvents. Thus, in this solvent system, the association or aggregation of $\alpha$-helices ${ }^{4-6}$ is of interest. In a previous paper, ${ }^{6}$ we investigated the effect of the helicogenic solvents on the packing of $\alpha$-helices in PMDG films. As helicogenic solvents, chloroform, DCM, 1, 2-dichloroethane (EDC), and DMF were used. The results of the X-ray measurements showed that PMDG films cast from chloroform or 
DCM solution had a well ordered structure consisting of $\alpha$-helices packed on a regular hexagonal lattice, while PMDG films cast from the DMF solution showed far less crystallinity. Furthermore, the circular dichroic measurements showed that only those films cast from EDC solutions maintained a uniform cholesteric twisted structure. This film showed a different X-ray diffraction pattern from that of former films. In this paper, ${ }^{6}$ the three phases prepared from chloroform (or DCM), DMF, and EDC solutions, were designated phase $C$, phase $I$, and phase $M$, respectively.

The formation of a film from a solution is a complex process in which the concentration of polypeptide increases until a solid is formed. It is well known that the cholesteric liquid crystal structure is formed in a high concentration range of polypeptide solutions. ${ }^{7}$ Thus, the film formation from a solution should be discussed in relation to this particular structure. In consideration of the nature of cholesteric liquid crystal structure, that is, the concentration dependence of cholesteric pitch, it is easy to assume that this cholesteric structure is retained in the final films. Thus, only the films cast from EDC solutions maintain the uniform cholesteric twisted structure.

The overall morphology and crystallinity of PMDG films may have an important effect on mechanical properties. Recently, the mechanical properties of PMDG films have been discussed on the basis of the molecular motion of $\alpha$-helices. ${ }^{4,6-8}$ Sakamoto, et al. ${ }^{8}$ have found two kinds of dispersions in the higher temperature range for drawn fibers of PMDG. They concluded that a dispersion at $160^{\circ} \mathrm{C}$ may be associated with the migrational slip of $\alpha$-helices in the disoriented region and a dispersion at $180^{\circ} \mathrm{C}$ with the mutual slip of the neighboring crystallites and/or the mutual slip of $\alpha$-helices in the crystalline regions. In view of the molecular motion of $\alpha$-helices, the mechanical properties have been extensively discussed by Kajiyama, et $a l^{4}$ It was also reported that two kinds of viscoelastic crystalline relaxation phenomena are observable in the temperature range from 140 to $170^{\circ} \mathrm{C}$ for PMDG films cast from the EDC solution, and from 180 to $190^{\circ} \mathrm{C}$ for films cast from the chloroform solution. They concluded that these relaxation mechanisms are associated with shear deformation in the interhelix region of the crystalline phase represented by migrational slip or rotational motion of $\alpha$-helices and also with the tensile or bending deformation of $\alpha$-helices, respectively. On the other hand, Wilkes, et al., ${ }^{5}$ reported that $\tan \delta$ loss peak at $145^{\circ} \mathrm{C}$ is characteristic of films having significant $\beta$-chain conformation. At a higher temperature range, two types of mechanical dispersions have been observed. A previous paper $^{6}$ reported that three mechanical losses were observed above $100^{\circ} \mathrm{C}$. They were named $\alpha_{1}$ dispersion (at $160^{\circ} \mathrm{C}$ ), $\alpha_{2}$ dispersion (at $190^{\circ} \mathrm{C}$ ), and $\alpha_{3}$ dispersion (above $200^{\circ} \mathrm{C}$ ) in order of increasing temperature. Comparing the mechanical dispersions with structure, it was assumed that $\alpha_{1}, \alpha_{2}$, and $\alpha_{3}$ dispersion are caused by molecular relaxation in phase $\mathrm{I}$, phase $\mathrm{C}$, and phase $\mathbf{M}$, respectively.

The primary purpose of this paper is to clarify the casting history of films and obtain detailed information on the superstructure of cast films, and secondly, to explain the mechanical properties in relation to a well-defined superstructure. The examinations of the structure and mechanical properties were made for a series of PMDG films cast from DCM-DMF solutions and chloroform-DMF solutions. Additional investigations were also made for PMDG films cast from $m$ cresol solutions at various temperatures and for the PMDG-PMLG blend films cast from chloroform solutions.

\section{EXPERIMENTAL}

\section{Materials}

PMDG $\left(M_{v}=100,000\right)$ and PMLG $\left(M_{v}=\right.$ 120,000) samples were kindly supplied by Ajinomoto Co., Ltd., Japan.

Films of PMDG were prepared from DCMDMF solutions and chloroform-DMF solutions at room temperature. PMDG $(1 \mathrm{~g})$ was first dissolved in $30-\mathrm{m} l \mathrm{DCM}$ (or $30-\mathrm{m} l$ chloroform) solvent, and then a certain quantity $(\mathrm{X} \mathrm{m} l)$ of DMF was added to this solution. The film cast from this solution was termed $\mathrm{F}-\mathrm{X}$ (or $\mathrm{S}-\mathrm{X}$ ), where $\mathrm{X}$ is the quantity of DMF solvent. It should be noted that PMDG-DMF solution forms a thermally reversible gel upon standing at room temperature. It was also found that PMDG in DCM (or chloroform)-DMF solutions forms a gel as evaporation of the solvent proceeds since the volatile DCM (or chloroform) solvent is allowed to 
evaporate more rapidly than the DMF solvent. Thus, some films were prepared after the gel formation of solutions in different concentrations depending on the quantity of DMF.

PMDG films were also prepared from $m$-cresol solutions at various temperatures in vacuo. Casting temeperature were $50,60,70,80$, and $90^{\circ} \mathrm{C}$, and the casting period was from 4 to 10 days. The films in this system were termed M-X, X being the casting temperature.

PMDG-PMLG blend films were cast from chloroform solutions at room temperature. Six blend films with a different L-component to Dcomponent ratio were prepared. Racemic films were also prepared from EDC solutions and DMF solutions. For the preparation of PMDGPMLG solutions, the PMDG solution and PMLG solution were mixed. Then the DMF solutions, but not the chloroform or EDC solutions, were found to form a white rigid gel.

Films were cast on a glass plate. After being dried, the films were immersed in methyl alcohol for $1-2$ days in order to remove any trace of solvent retained in the films, and then dried again in vacuo. From IR measurements, it was found that all films consisted of $\alpha$-helical molecules.

\section{Methods}

X-ray measurements were carried out with a Rigaku Denki X-ray generator (Ni-filtered $\mathrm{CuK} \alpha$ Radiation). X-ray photographs were taken with a flat-plate camera. In order to calibrate the camera distance, the samples were covered with silicon powder. A symmetrical-reflection technique was employed to record the diffraction intensity curves, which were detected by a scintillation counter with a pulse-height analyzer. Intensities were counted at $0.05^{\circ}$ intervals in Bragg angle $(2 \theta)$ and the counting period was $80 \mathrm{sec}$.

Circular dichroism was measured by using a JASCO Automatic Recording Spectropolarimeter Model J-5. The incident light was cast at right angle to the film surface.

Photomicrographs of samples were taken with an Olympus-POM optical polarizing microscope.

Dynamic mechanical measurements were performed with a Vibron viscoelastometer (Toyo Measuring Instrument Co., Ltd.), in the temperature range from -50 to $270^{\circ} \mathrm{C}$ at frequencies of 110,11 , and $3.5 \mathrm{~Hz}$.

\section{RESULTS AND DISCUSSION}

\section{Casting Process of PMDG Films}

X-ray diffraction patterns for a series of PMDG films cast from DCM-DMF solutions are shown in Table I. According to these patterns, the structures of films were classified into three types. The film of the first type made from a DMF rich solution, shows only one diffuse reflection corresponding to spacing of about $11 \AA$. The films of the second type are from $\mathrm{F}-0.4$ to $\mathrm{F}-0.1$, and show a characteristic diffraction pattern which was observed for the cast film from the EDC solution. ${ }^{6}$ Furthermore, since these films have a cholesteric color thus confirming a uniform cholesteric twisted structure, they are classified into the same type as the films cast from EDC solution. ${ }^{9}$ For the films of the third type which are F-0.025 and F-0.01, the major part of reflections are fully explained by a hexagonal unit cell except for two reflections of 11.2 and $8.1 \AA$. Thus, from the DCM-DMF solutions, phase I, phase $\mathrm{M}$, and phase $\mathrm{C}$ as mentioned in a previous paper, ${ }^{6}$ could be systemati-

Table I. X-ray diagrams of PMDG films cast from DCM-DMF solutions

\begin{tabular}{|c|c|c|c|c|c|c|c|}
\hline $\mathrm{F}-10$ & $F-2$ & $\mathrm{~F}-1$ & $\mathrm{~F}-0.4$ & $F-0.1$ & $\mathrm{~F}-0.06$ & $\mathrm{~F}-0.025$ & $\mathrm{~F}-0.01$ \\
\hline \multirow{9}{*}{10.9} & & & 12.1 & 12.2 & & & \\
\hline & 11.2 & 11.3 & & & 10.9 & 11.3 & 11.2 \\
\hline & & & 10.5 & 10.6 & & 10.3 & 10.3 \\
\hline & & & & & & & 8.12 \\
\hline & 7.81 & 7.88 & 7.79 & 7.84 & & & \\
\hline & & & & & & 5.99 & 5.94 \\
\hline & & & & & & 5.11 & 5.10 \\
\hline & & & & & & 4.45 & 4.47 \\
\hline & & & & & & 3.83 & 3.87 \\
\hline
\end{tabular}




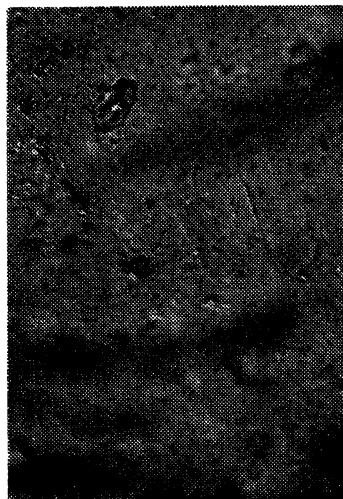

(A)

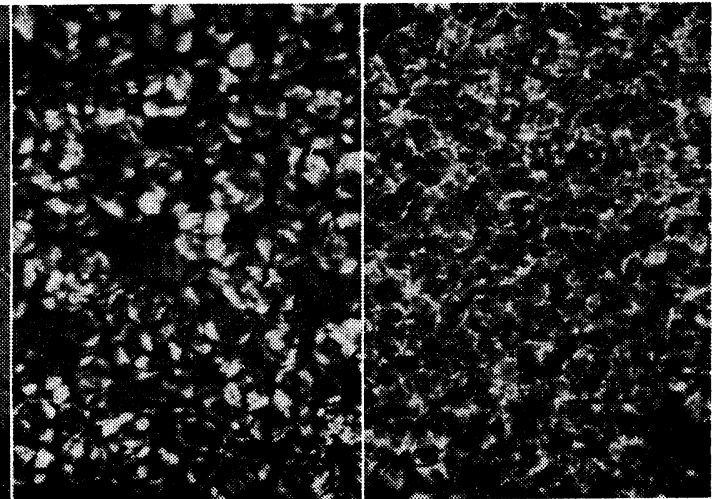

(B)
(C)

Figure 1. Optical photomicrographs taken with cross polaroids $(\times 400)$ of $(\mathrm{A})$ F-10, (B) F-2, and (C) F-0.1 cast from DCM-DMF solutions.

cally prepared depending on the DMF content of the mixed solvent.

By a detailed observation of the X-ray pattern in Table I, it was also found that the X-ray pattern successively changes in a series of films from F-10 to $F-0.1$; the reflections of $12.2,10.5$, and $7.8 \AA$ gradually appear, and that there is a remarkable difference between the structures of $\mathrm{F}-0.1$ and $\mathrm{F}-$ 0.025. The former change in the X-ray pattern does not seem due to a gradual change of the structure from phase I type to phase $M$ type, but rather to the successive change in the relative ratio of the area of phase $M$ to the area of phase $I$ from the following observation of micrographs.

Polarized micrographs of $\mathrm{F}-10, \mathrm{~F}-2$, and $\mathrm{F}-0.1$ are shown in Figure 1. F-10 has a uniform area which seems to be phase I, and no other structure is observed. F-2 consists of phase $I$ and phase $M$, since it is observed that the spherulites of phase $M$, showing clearly the Marthese cross, are dispersed in phase I. This indicates that the morphological structure of the solution was retained to F-2 during the solidification of the solution in which the cholesteric liquid crystal phase as a sphere and the isotropic phase coexist. ${ }^{7} \quad$ Judging from the similarity in X-ray patterns of $F-2$ and $F-1$, this coexistence of phase $I$ and phase $M$ is also presumed for $\mathrm{F}-1$ although this was not clearly observed by microscope. In Figure 1 (C), it is likely that F-0.4 or F-0.1 does not consist of a uniform domain with a cholesteric twisted structure but rather may consist of a poly-domain structure of $10-20$ microns. This domain structure seems to be supported from the scattering pattern of the small angle light scattering made with a helium neon laser. ${ }^{10}$ This film showed a clover leaf pattern in crossed polaroids, confirming the existence of anisotropic spheres or disks. Their size was determined from the maximum intensity of scattering $^{11}$ to be also $10-20$ microns. Thus, the macro-structure for these films including $\mathrm{F}$ $0.06-F-0.01$ may be schematically illustrated in Figure 2 and, in conclusion, for the films of F-10 -F-0.1, the relative ratio of the area of phase I to the area of phase $M$ decreases with a decrease in the quantity of the DMF solvent added to the DCM solution. The discussion for the abrupt change between the structure of F-0.1 and 0.025 will be made below.

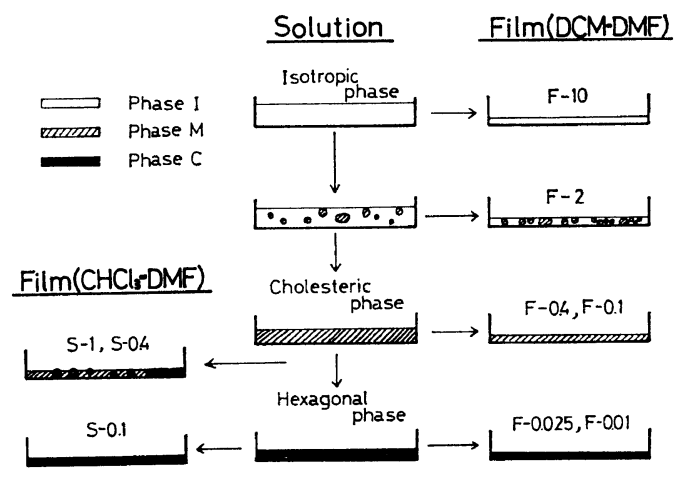

Figure 2. Schematic representation of casting history and macro-structure of PMDG cast films from DCM-DMF solutions and chloroform-DMF solutions. 


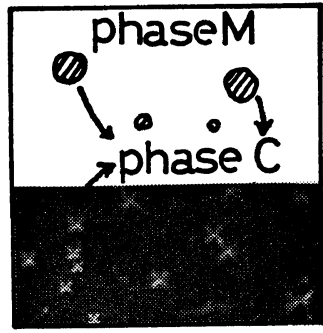

(a) $\mathrm{S}-1$

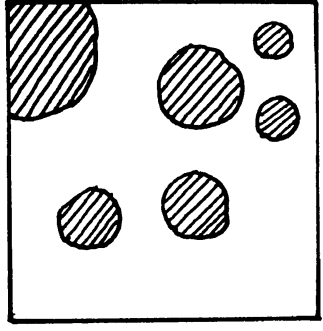

(b) S-0.4

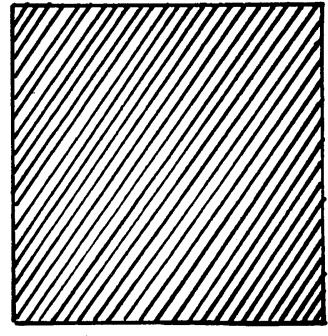

(c) $\mathrm{S}-0.1$

Figure 3. Sketch of (a) S-1, (b) S-0.4, and (c) S- 0.1 cast from chloroform-DMF solutions. In photograph of (a) taken with cross polaroids, the spherurites in $\mathrm{S}-1$ film show clearly the Martese cross.

It is well known that PMDG molecules form a cholesteric liquid crystal structure in concentrated solution. $^{7}$ It has been observed by microscope that the chloroform or DCM solutions form the uniform cholesteric liquid crystal structure up to the concentration of about $40 \mathrm{wt} \%{ }^{6}{ }^{6}$ From X-ray and circular dichroic (CD) measurements, however, it was found that no films cast from chloroform or DCM solutions maintain the cholesteric twisted structure. ${ }^{9}$ Thus, the cholesteric liquid crystal structure should collapse at a still higher concentration of chloroform or DCM solutions. In order to confirm this collapse of the cholesteric liquid crystal, the structure of films prepared from chloroform-DMF mixtures was examined. The structures of the films of S-10 and S-2 were the same as those for $\mathrm{F}-10$ and $\mathrm{F}-2$ in the DCM-DMF system, respectively. Interesting results were obtained for $\mathrm{S}-1$ and $\mathrm{S}-0.4$. As sketched in Figure 3, these films consist of two phases; one phase can be visibly observed as spheres dispersed in the other phase. From X-ray measurements, the structure of spherulites was found to be hexagonal with the same unit cell as in the film cast from chloroform solution, while the structure of the latter phase was the same as that of the film cast from EDC solution. The latter phase does also show the cholesteric color and the cholesteric band in the CD curve (see Figure 4), ${ }^{9,12,13}$ confirming that it is phase M. Furthermore, Figure 3 shows that the area of phase $\mathrm{M}$ becomes small in $\mathrm{S}-0.4$, and disappears in S-0.1. From these results, it is obvious that the molecular rearrangement of $\alpha$-helices from a twisted array to one that is parallel, occurs in the chloroform concentrated solutions. On the other hand, the abrupt change between

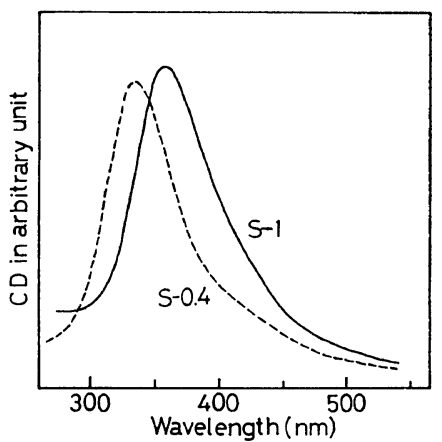

Figure 4. Circular dichroism of phase $\mathrm{M}$ in $\mathrm{S}-\mathbf{1}$ (-) and S-0.4 (-----).

the X-ray diffraction pattern of $\mathrm{F}-0.1$ and that of F-0.025 in the DCM-DMF system, can also be explained by such a collapse of the cholesteric twisted structure, although the formation of spherulites was not observed in this system.

By Ishikawa, et al., ${ }^{14}$ PMDG films with the isolated spherulites were prepared from well-aged (for several months) solutions in chloroform. This aging time was one of the main factors in making the spherulites; a short period ( $1-6$ months) led to positive spherulites, while by a long period, (6-10 months), negative spherulites were obtained. From results on the preparation of spherulites, it was found that the spherulites $(1-3 \mathrm{~mm}$ diameters) could be obtained in a much shorter period (a few days) from the chloroform-DMF solvent as well as from the bromoform solvent. ${ }^{15}$ Thus, the formation mechanism of these spherulites is considered to be different from that reported by Ishikawa, et al., ${ }^{14,16}$ Furthermore, it is of interest that sherulites in the chloroform-DMF solvent 
system were induced from the cholesteric liquid crystal phase. A structural examination of the spherulites will be made in near future.

\section{Structure of Phase I, Phase $M$, and Phase C}

As mentioned above, the structures of PMDG cast films are categorized into three types, phase I, phase $\mathrm{M}$, and Phase C. The preparation of these phases is shown in Table II.

Table II. Preparation of films with phase I, phase $M$, and phase $C$

\begin{tabular}{ccc}
\hline Phase I & \multicolumn{1}{c}{ Phase $\mathrm{M}$} & Phase $\mathrm{C}$ \\
\hline DMF cast film & EDC cast film & $\begin{array}{c}\text { Chloroform cast } \\
\text { film } \\
\text { DCM cast film } \\
m \text {-cresol cast } \\
\text { film }\end{array}$ \\
$\sim \mathrm{F}-10^{\mathrm{a}}$ & $\mathrm{F}-0.4, \mathrm{~F}-0.1$ & F-0.025 \\
$\sim \mathrm{S}-10^{\mathrm{a}}$ & $\mathrm{S}-1, \mathrm{~S}-0.4^{\mathrm{b}}$ & $\mathrm{S}-0.1 \sim$ \\
\hline
\end{tabular}

${ }^{a} \mathrm{~F}$ and $\mathrm{S}$ indicate films cast from DCM-DMF and chloroform-DMF mixtures, respectively.

b These films also have phase $\mathrm{C}$ as shown in Figure 3.

Phase I was obtained after the gel formation of the isotropic solution in DMF. During the evaporation of solvent, the syneresis of the DMF solvent was observed. The gel formation is considered to be attributed to the fibrilar association of $\alpha$ helices. ${ }^{17}$ From the results of X-ray diffraction pattern of phase I, the associations of $\alpha$-helices and/or microfibrils exhibit an extremely poor order.

Phase $M$ was prepared from the EDC, DCMDMF mixture and chloroform-DMF mixture, where the DMF content in the mixture is an important factor in the preparation of phase $\mathrm{M}$ as mentioned above. The structure of phase $M$ is remarkably different from that of the other phases and consists of the cholesteric twisted arrangement of $\alpha$-helices. This solid cholesteric structure arises from the solidification of a cholesteric liquid crystal structure observed in concentrated solutions. The circular dichroic results shown in Figure 4 clarify the nature of this structure. The cholesteric twisted structure is well known to consist of rotating layers of the rod-like molecules and to be characterized by the presence of a helical twist of uniform pitch. ${ }^{7}$ In a previous paper, ${ }^{9}$ we reported that the pitch of the helical twist is from 250 to $400 \mathrm{~nm}$ and that the twisted angle per rotating layer is from 1 to 2 degrees. The color com- monly observed in this type of film is structural and is due to the selective reflection of one component of the circularly polarized light. This helicoidal structure is noticeable in biological analogues of cholesterics ${ }^{18,19}$ and from optical properties. $^{12,13}$

Phase C, that is the hexagonal crystalline phase, was prepared from chloroform or DCM solutions. This phase can be also obtained from $m$-cresol solutions as mentioned below. From the microscopic observation of chloroform solutions ${ }^{6}$ and from the observation of the morphology of films cast from chloroform-DMF solvents, it became clear that phase $\mathrm{C}$ occurs as a result of a collapse of the cholesteric liquid crystal structure. In a molecular rearrangement from a twisted array to a parallel one, the hexagonal crystalline phase should be divided into small mosaic blocks retaining more or less a trace of the cholesteric helical arrangement. This speculation seems to be supported by examinating the structure of the films from $m$-cresol solutions.

\section{Influence of Casting Temperature on Structure of $P M D G$ Films}

Phase $\mathrm{C}$ was also prepared from $m$-cresol solutions. Since $m$-cresol has a high boiling point $\left(202^{\circ} \mathrm{C}\right)$, the influence of the casting temperature on the structure of PMDG films can be examined. Table III shows the X-ray diagrams of the films

Table III. X-ray diagrams of PMDG films cast from $m$-cresol solutions at various temperatures

\begin{tabular}{|c|c|c|c|}
\hline \multicolumn{3}{|c|}{ (2) } & \multirow{2}{*}{$\frac{(\AA)}{\mathrm{M}-90}$} \\
\hline Index ${ }^{a}$ & $M-50$ & $M-70$ & \\
\hline 100 & 10.36 & 10.34 & 10.34 \\
\hline 110 & 5.99 & 5.99 & 5.96 \\
\hline 112 & 5.51 & 5.52 & 5.52 \\
\hline 200 & 5.19 & 5.17 & 5.16 \\
\hline 105 & 4.83 & 4.84 & 4.84 \\
\hline 115 & 4.03 & 4.04 & 4.04 \\
\hline 210 & 3.90 & 3.90 & 3.88 \\
\hline
\end{tabular}

a Index is based on the hexagonal unit cell.

cast from $m$-cresol solutions at various temperatures, and Figure 5 (B) shows the X-ray diffraction photograph of $M-90$. For all films, the reflections are indexed on the basis of the hexagonal lattice with the same dimensions as for the film 


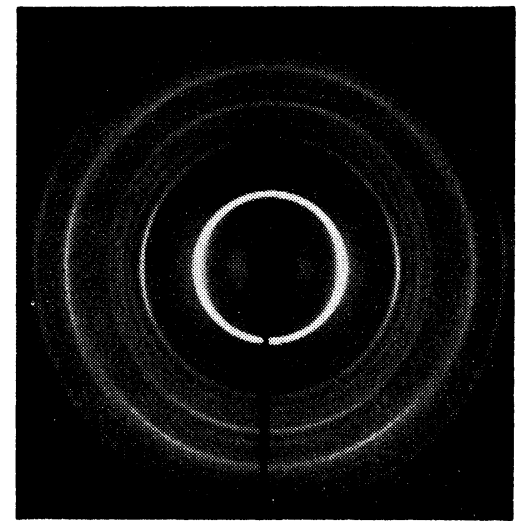

(A)

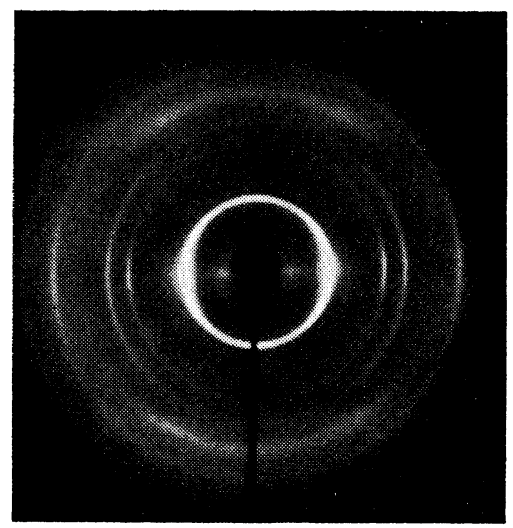

(C)

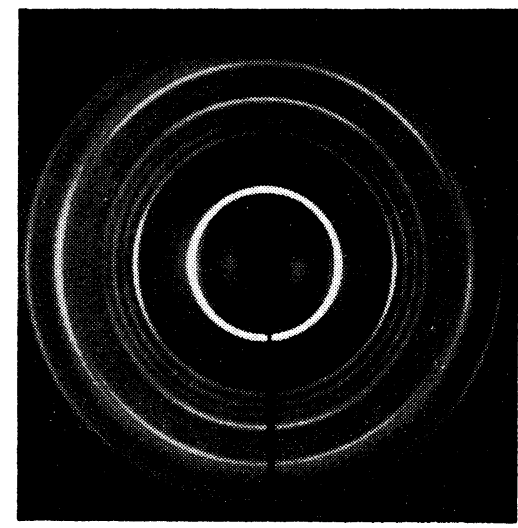

(B)

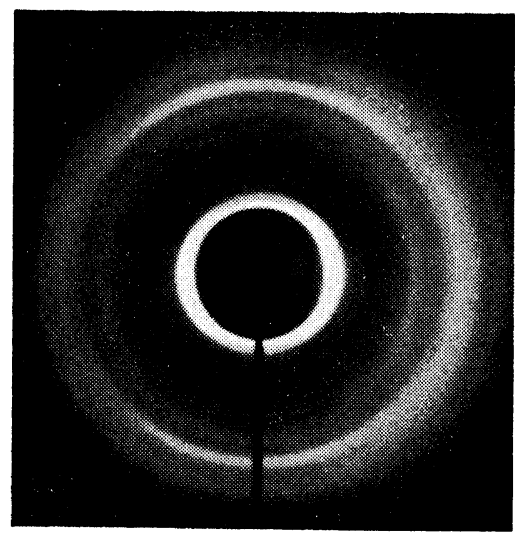

(D)

Figure 5. X-ray photographs of (A) PMDG film cast from chloroform, (B) PMDG film cast from $m$-cresol at $90^{\circ} \mathrm{C}$, (C) PMDG-PMLG racemic film cast from chloroform, and (D) PMDG-PMLG racemic film cast from EDC. The X-ray beam is parallel to the film surface.

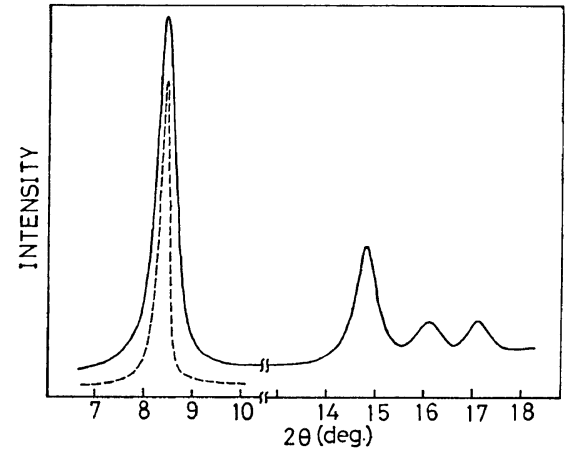

Figure 6. X-ray diffraction profile of the equatorial reflection of M-50 (-) and M-90 (----) measured by symmetrical-reflection technique. cast from chloroform solution. The molecular packing of $\alpha$-helices, thus, were not affected by the casting temperature.

However, it was found that the breadth of the (100) reflection was very different in $\mathrm{M}-50$ and M-90 (see Figure 6). The symmetrical-reflection method is profitable for obtaining a profile of the equatorial reflections of cast films having a planar orientation. $^{20}$ But this method has some difficulties in the range of a small diffraction angle. Figure 7 shows the plots of the integral breadth of the $(100)$ reflection against the casting temperature, where the instrumental broadening was corrected by use of the (111) reflection of the silicon powder poured on the film surface. The integral breadth of the (100) reflection decreases with an increase in the casting temperature. From the X-ray line 


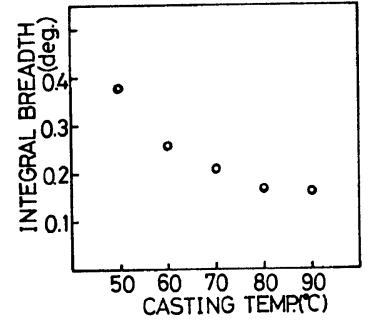

Figure 7. Plots of the integral breadth of the (100) reflection for $m$-cresol cast films against the casting temperature.

profile data, the crystallite size and/or lattice distortion information can be found. ${ }^{20,21}$ The line profiles of size and distortion broadenings were assumed to be Cauchy. The apparent crystallite size, $L$, and the relative distance fluctuation of the $(100)$ spacing, $g=\Delta d / d$, were estimated from the plots of the integral breadth $v s$. square of the order of reflection using the (100), (200), and (300) reflections; $L=270 \AA$ and $g=0.02$ for $\mathrm{M}-50$, and $L=870 \AA$ and $g=0.017$ for $M-80$. Thus, the change of the integral breadth of the (100) reflection in Figure 7 is due mainly to the change of crystallite size, and it is obvious that the crystallite size in a direction normal to the (100) planes increases from about 300 to $900 \AA$ with an increase in the casting temperature.

Since the $m$-cresol solution also forms the cholesteric liquid crystal in high concentration, ${ }^{7}$ the casting process of PMDG films cast from $m$-cresol solutions is the same as that of the chloroform cast film. In this regard, it is interesting that the casting temperature has a significant effect on the crystallite size. It is known that in the poly $(\gamma$ benzyl glutamate) solution system, the cholesteric pitch increases with increasing temperature. ${ }^{22}$ This increase in cholesteric pitch is attributed mainly to a decrease in the twisted angle per rotating layer. Judging from this general phenomena of the temperature dependence of pitch, it is likely that the increase of crystallite size in $\mathrm{m}$ cresol cast films was caused by an increase in the cholesteric pitch in the high concentrated solution. In consideration of the occurrence of the hexagonal phase from the cholesteric phase (Figure 3), the effect of the cholesteric pitch on the crystallite size may be supposed; the crystallites or small

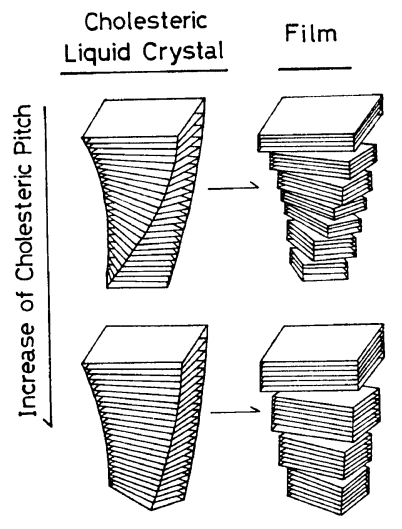

Figure 8. Illustration of the appearance of the small mosaic crystallites in phase $\mathrm{C}$ and the dependence of the crystallite size on the casting temperature.

blocks of comparably small size may yield as a result of a molecular rearrangement of the $\alpha$ helices from the twisted array to the parallel one and their size becomes larger as the cholesteric pitch increases. In Figure 8, these phenomena are tentatively illustrated. By this view point of the structutre, it is of interest to examine the mechanical properties, since the mechanical properties of these films are different from each other (see later, e. g., Figure 17).

\section{Structure of Blend Films of PMDG and PMLG}

Table IV shows the X-ray diffraction patterns for the blend films cast from chloroform solutions. The equatorial reflections are the same for all the films and show that the $\alpha$-helical molecules in lateral directions are packed into the hexagonal lattice with the same unit cell as for PMDG film cast from chloroform solution. On the other hand, the layer line reflections gradually disappear as the ratio of $\mathrm{D}$ - and $\mathrm{L}$-component reaches $1: 1$, and are replaced by layer line streaks (see Figure 5 (c)). Thus, for the blend films cast from chloroform, a random location of $\mathrm{D}$ - and $\mathrm{L}$-molecules on the net point, but not a regular pairing of $\mathrm{D}$ - and L-molecules, seems likely. ${ }^{23,24}$

The X-ray diffraction pattern of the racemic mixture prepared from EDC solution or DMF solution, was different from that of the racemic mixture from the chloroform solution (Table IV and Figure $5(\mathrm{D}))$. The reflections of a larger spacing than $5 \AA$ in Table IV, were considered to 
Structure and Mechanical Properties of PMG Films. II.

Table IV. X-Ray diagrams of PMDG-PMLG blend films

( $)$

\begin{tabular}{|c|c|c|c|c|c|c|c|}
\hline \multicolumn{7}{|c|}{ Chloroform cast films } & \multirow{2}{*}{$\frac{\text { EDC cast film }}{1: 1}$} \\
\hline Index ${ }^{a}$ & $\begin{array}{c}\text { PMDG:PMLG } \\
10: 1\end{array}$ & $8: 1$ & $6: 1$ & $6: 1$ & $2: 1$ & $1: 1$ & \\
\hline 100 & 10.38 & 10.36 & 10.38 & 10.40 & 10.40 & 10.40 & 11.5 \\
\hline 110 & 5.94 & 5.94 & 5.94 & 5.95 & 5.94 & 5.96 & 10.4 \\
\hline 112 & 5.43 & 5.53 & & & & & 8.9 \\
\hline 200 & 5.12 & 5.16 & 5.15 & 5.14 & 5.18 & 5.16 & 7.7 \\
\hline \multirow[t]{4}{*}{105} & 4.78 & 4.80 & 4.82 & 4.82 & & & 6.8 \\
\hline & 4.49 & 4.48 & 4.49 & & & & 5.7 \\
\hline & & 4.4 & 4.4 & 4.4 & 4.4 & 4.5 & 5.2 \\
\hline & & (diff.) & (diff.) & (diff.) & (diff.) & (diff.) & 5.1 \\
\hline 115 & 4.02 & 4.01 & 4.02 & & & & 4.9 \\
\hline 210 & 3.86 & 3.89 & 3.88 & 3.86 & 3.93 & 3.90 & 4.4 \\
\hline \multirow[t]{4}{*}{300} & 3.41 & 3.44 & 3.46 & & & & (diff.) \\
\hline & & & & & & & 4.2 \\
\hline & & & & & & & 4.0 \\
\hline & & & & & & & 3.5 \\
\hline
\end{tabular}

${ }^{a}$ Index is based on the hexagonal unit cell.

be equatorial, since the reflections on the 2 nd and 3rd layer lines may be broadened by the rotational and/or translational disorder of $\alpha$-helical molecules as implied from the observation of the 5th layer line streaks corresponding to about $4.4 \AA$. These reflections can be fairly well explained on the basis of the two-dimensional unit cell with $a=$ $23.1 \AA, b=10.4 \AA$, and $\gamma=100^{\circ}$, showing that two molecules are contained in a one unit cell. These results, together with the fact that the racemic compound has been formed in the DMF solution of PMDG and PMLG mixture, ${ }^{25}$ indicate that there exists a special interaction between $D$ - and L-molecules in the films cast from EDC solution or DMF solution, although, at present, it cannot be clarified as to what sort of interaction exists.

\section{Mechanical Properties of PMDG Films and PMDG - PMLG Blend Films \\ $\alpha_{1}$ and $\alpha_{3}$ Dispersion. For a series of films from} F-10 to F-0.1 in the DCM-DMF solvent system, the mechanical properties were examined. Figures 9 and 10 show mechanical properties at 110 and $3.5 \mathrm{~Hz}$, respectively. For these films, the mechanical dispersions above $100^{\circ} \mathrm{C}$ are described by $\alpha_{1}$ and $\alpha_{3}$ dispersions. From Figure 9, it is seen that the mechanical properties of $F-10$ and $F-0.1$ are very similar to those of cast films from the DMF solution and EDC solution, ${ }^{6}$ respectively, as ex- pected from the similarity of the structure. The $\alpha_{1}$ loss tangent has a maximum peak height for F10. The peak height gradually lowers for $F-2$ and $\mathrm{F}-1$, and then becomes minimum for $\mathrm{F}-0.1$. Such a decrease of peak height of the $\alpha_{1}$ loss tangent closely corresponds to the decrease of phase I region. Similar phenomena were observed by

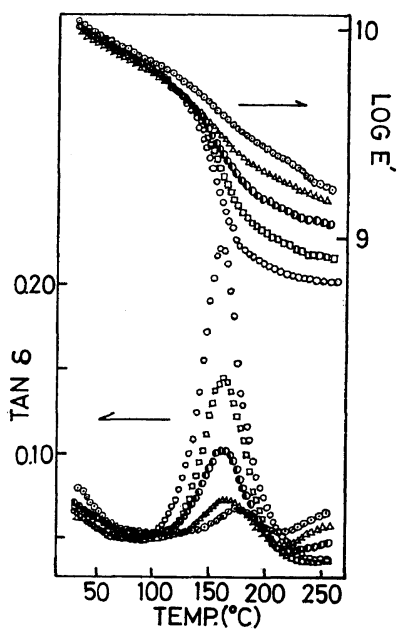

Figure 9. Plots of $\log \mathrm{E}^{\prime}$ and $\tan \delta$ vs. temperature for F-10 (O), F-2 ( $\square), F-1(0), F-0.4(\triangle)$, and F-0.1 (๑) cast from DCM-DMF solutions. Data obtained at $110 \mathrm{~Hz}$. 


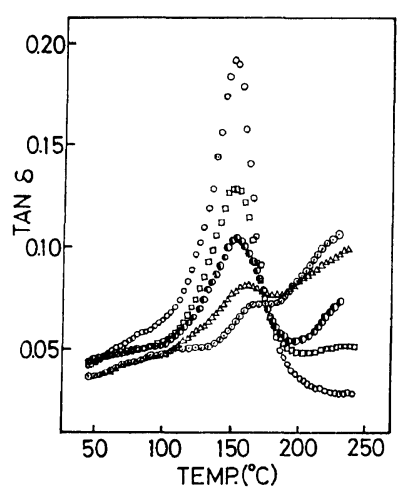

Figure 10. Plots of $\tan \delta$ vs. temperature for $\mathrm{F}-10$

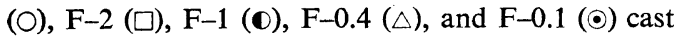
from DCM-DMF solutions. Data obtained at $3.5 \mathrm{~Hz}$.

Sakamoto, et al., ${ }^{8}$ i.e., with an increase in the degree of orientation by the creep deformation at $150^{\circ} \mathrm{C}$, the crystallinity of PMDG fibers increases and the peak height of the tan $\delta$ loss at $160^{\circ} \mathrm{C}$ lowers, and it is concluded that this loss tangent peak may be attributed to a mutual slip of $\alpha$ helices in the disoriented region. This correspondence of structure and the peak height of $\alpha_{1}$ loss tangent, together with the results by Sakamoto, et al. ${ }^{8}$ show that the $\alpha_{1}$ dispersion at $150-160^{\circ} \mathrm{C}$ can be associated with some kinds of molecular motion of $\alpha$-helices in phase I which showed a far less degree of crystallinity in the X-ray diagram. It was noted that the $\alpha_{1}$ loss peak can be observed even in F-0.4 and F-0.1 with the cholesteric twisted structure. This implies that a phase similar to phase I exists between the domains of

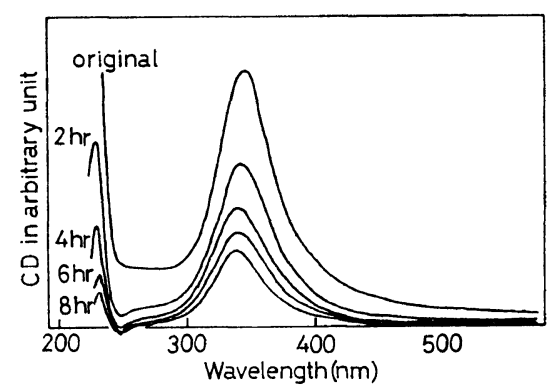

Figure 11. Dependence of the circular dichroic curve on annealing time for phase $\mathrm{M}$ in $\mathrm{S}-0.4$ annealed at $200^{\circ} \mathrm{C}$.

phase $M$ as can be seen in Figure 1 (C).

In Figure 10, the $\alpha_{3}$ loss tangent observed above $200^{\circ} \mathrm{C}$ appears gradually with an decrease in the magnitude of the $\alpha_{1}$ loss tangent and becomes remarkable in F-0.4 and F-0.1, although the peak maximum was not found in the temperature range up to $260-270^{\circ} \mathrm{C}$. This $\alpha_{3}$ loss tangent was also observed in the EDC cast film and in the phase $\mathrm{M}$ area of S-0.4 (see later Figure 14). From the fact that the $\alpha_{3}$ loss tangent can be observed vividly in the films with a cholesteric twisted structure, this loss tangent may be attributed to the relaxation in phase M. It has been observed that the circular dichroic curve due to the cholesteric twisted structure gradually disappeared by heat treatment above $200^{\circ} \mathrm{C},{ }^{9}$ whereas, in the temperature range of 20 $180^{\circ} \mathrm{C}$, the circular dichroic curve shows only a reversible peak shift brought on by linear expansion of the molecular distance. ${ }^{26}$ An example of the annealing effect is shown in Figure 11, indicat-

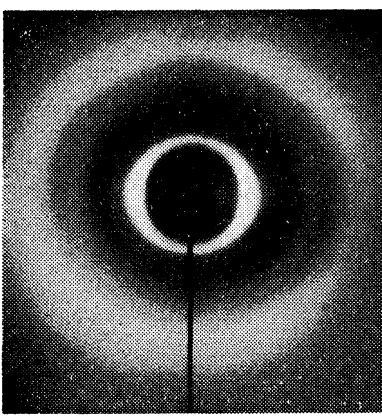

(A)

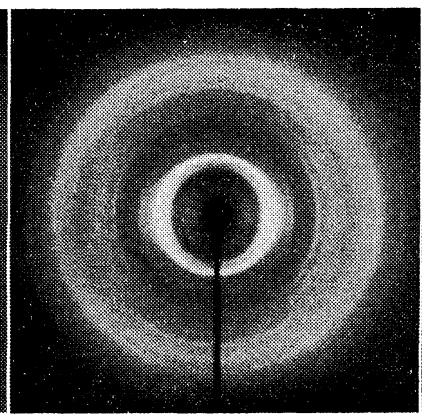

(B)

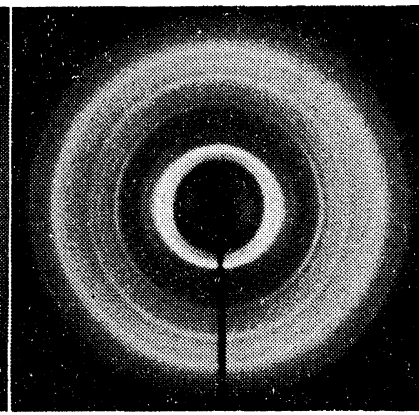

(C)

Figure 12. X-ray photographs for phase M: (A), original; (B), annealed at $200^{\circ} \mathrm{C}$ for $2 \mathrm{hr}$; (C), annealed at $220^{\circ} \mathrm{C}$ for $1 \mathrm{hr}$. 
ing the dependence of the circular dichroic curve on the annealing time for phase $\mathrm{M}$ of S- 0.4 annealed at $200^{\circ} \mathrm{C}$. Also Figure 12 shows the X-ray diffraction photographs for films annealed at 200 and $220^{\circ} \mathrm{C}$. The characteristic X-ray pattern (Figure 12 (A)) which may be attributed to the twisted structure, gradually changed to the hexagonal pattern by heat treatment above $200^{\circ} \mathrm{C}$. These results show that an appreciable molecular rearrangement of $\alpha$-helices may occur above $200^{\circ} \mathrm{C}$. Such a molecular rearrangement of $\alpha$-helices should be caused by an incoherent molecular motion between the individual $\alpha$-helices induced thermally in phase $M$, which seems to be associated with the $\alpha_{3}$ dispersion above $200^{\circ} \mathrm{C}$.

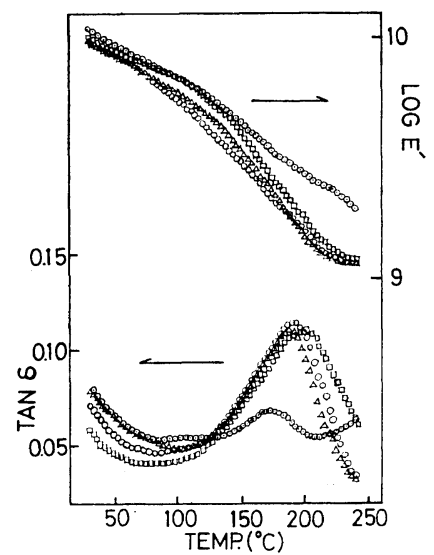

Figure 13. Plots of $\log \mathrm{E}^{\prime}$ and $\tan \delta$ vs. temperature for $F-0.06(O), F-0.025(\triangle)$, and $F-0.01(\square)$ cast from DCM-DMF solutions. The data for F-0.1 $(\odot)$, are shown in Figure 9 and here also. Data obtained at $110 \mathrm{~Hz}$.

$\alpha_{2}$ Dispersion. Figure 13 shows the mechanical properties for $\mathrm{F}-0.06, \mathrm{~F}-0.025$, and $\mathrm{F}-0.01$. By a comparison of Figure 9 with Figure 13, the remarkable differences are found to lie in the mechanical properties of F-0.1 and F-0.06. Further, for the films of $F-0.06, F-0.025$, and $F-0.01$, only the $\alpha_{2}$ dispersion is observed above $100^{\circ} \mathrm{C}$. Figure 14 shows the mechanical properties for phase $\mathrm{C}$ and phase $\mathrm{M}$ in $\mathrm{S}-0.4$. As expected from the similarity in structure, the property of phase $C$ in $\mathrm{S}-0.4$ is the same as that of the chloroform cast film and one of the phase $\mathrm{M}$ in $\mathrm{S}-0.4$ is similar to that of the EDC cast film. Thus, the $\alpha_{2}$ dispersion

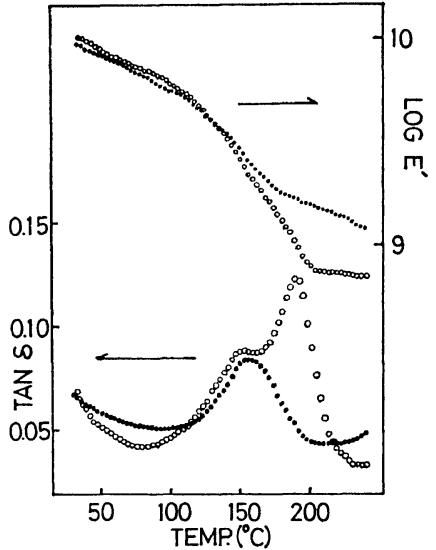

Figure 14. Plots of $\log \mathrm{E}^{\prime}$ and $\tan \delta$ vs. temperature for phase $\mathbf{C}(O)$ and phase $\mathbf{M}(\bullet)$ in $\mathbf{S}-\mathbf{0 . 4}$. Data obtained at $110 \mathrm{~Hz}$.

is commonly observed for phase $\mathrm{C}$ which shows a hexagonal diffraction pattern. Two relaxation mechanism for $\alpha_{2}$ dispersion can be discussed here in relation to the structure of phase $\mathrm{C}$.

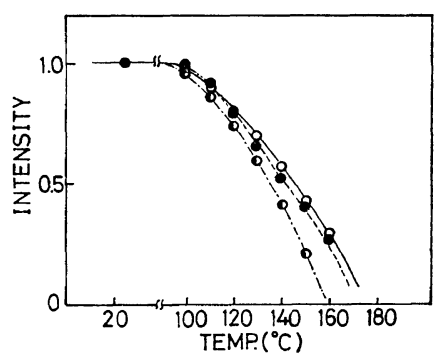

Figure 15. Temperature dependence of the intensities of $112(\bullet), 113(0)$, and $205(0)$ reflections for the oriented film prepared from concentrated solution in chloroform.

Figure 15 shows the temperature dependence of the intensities of the (112), (113), and (205) reflections for the oriented film prepared from concentrated solution in chloroform. In order to obtain the appreciable intensity of layer line reflection, the oriented film was neccessary. When the temperature is raised above $130-140^{\circ} \mathrm{C}$, the equatorial reflections remain sharp but the 2 nd, $3 \mathrm{rd}$, and 5 th layer lines become diffused, as seen in Figure 15. The disappearance of Bragg reflections on the layer lines shows that the librational 


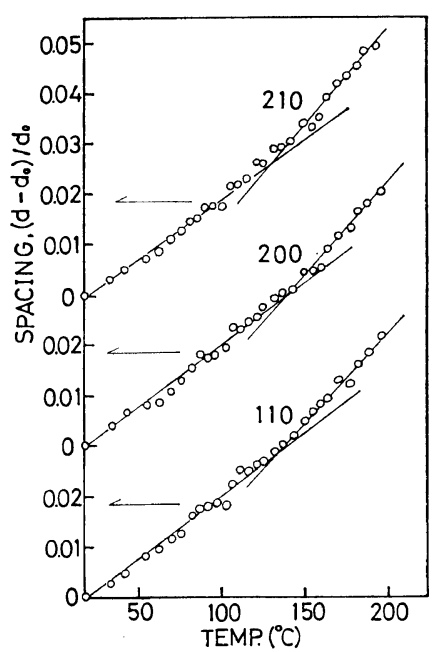

Figure 16. Temperature dependence of spacing of the (110), (200), and (210) reflections for PMDG films cast from chloroform solutions. $d_{0}$ is the value of $d$ at $20^{\circ} \mathrm{C}$.

motion of $\alpha$-helices about their long axes and the translational motion along them occur, ${ }^{27,28}$ while the molecules in lateral directions remain in regular hexagonal packing. In Figure 16, the spacings of the (110), (200), and (210) reflections for the chloroform cast film are plotted against temperature. The spacing-temperature plots exhibit a break at about $130^{\circ} \mathrm{C}$. In this film, it is reasonable to assume that this break results from the same origin as the disappearance of the reflection on layer lines as mentioned above. Thus, it is possible to attribute the $\alpha_{2}$ dispersion at $190^{\circ} \mathrm{C}$ to molecular motion such as that of $\alpha$-helices in phase C as predicted from the onset of librational and translational motions at $130-140^{\circ} \mathrm{C}$. Kajiyama, et al.,${ }^{4}$ also reported the association of $\alpha_{2}$ dispersion with the molecular motion of $\alpha$-helices in the hexagonal crystalline phase. From examination of the anisotropy of mechanical loss and the stability of the helix by X-ray and IR techniques, they have assumed that this dispersion was attributable to the tensile or bending deformation of $\alpha$-helices. Their results by X-ray technique are in agreement with our results.

The detailed observations of mechanical properties for PMDG films cast from $m$-cresol solutions and for a series of PMDG-PMLG blend films cast from chloroform solutions, however, do not seem to support the association of $\alpha_{2}$ dispersion with some kind of molecular motion of $\alpha$-helices in phase C. Figure 17 shows the mechanical loss for $m$-cresol cast films. In spite of the identity in molecular packing (see Table III), $\alpha_{2}$ loss peak temperature gradually shifts to a higher temperature with an increase in the casting tem-

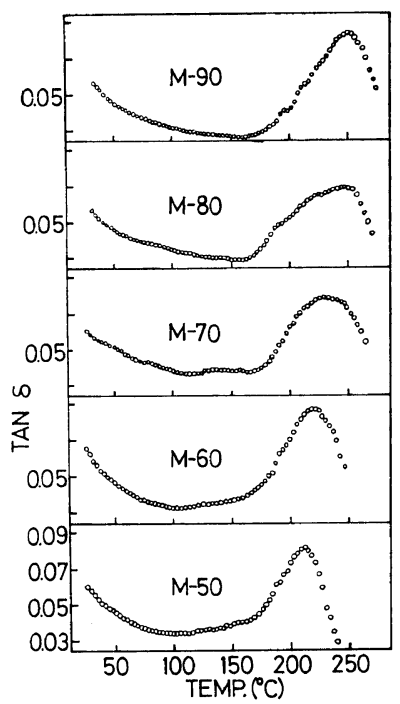

Figure 17. Plots of $\tan \delta v s$. temperature for PMDG films cast from $m$-cresol solutions at various temperatures. Data obtained at $110 \mathrm{~Hz}$.

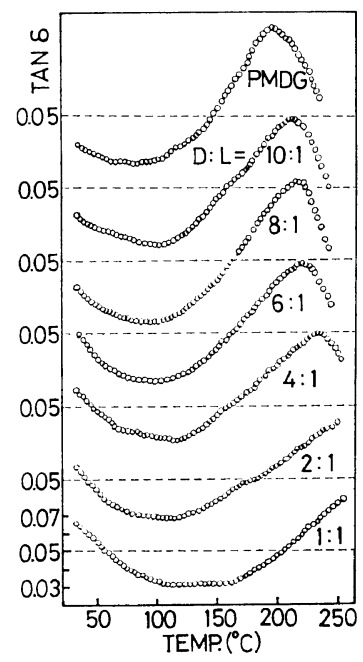

Figure 18. Plots of $\tan \delta$ vs. temperature for PMDG -PMLG blend films cast from chloroform solution. Data obtained at $110 \mathrm{~Hz}$. 


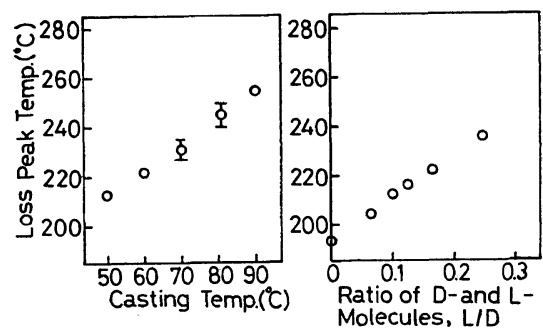

Figure 19. Plots of the $\alpha_{2}$ loss peak temperature $v s$. (A) the casting temperature for PMDG films cast from $m$-cresol solutions and (B) the ratio of $\mathrm{D}$ - and $\mathrm{L}$ molecules for PMDG-PMLG blend films cast from chloroform solutions.

perature. For a series of PMDG-PMLG blend films, it is also observed that the $\alpha_{2}$ peak temperature becomes higher as the ratio of $\mathrm{D}$ - and Lcomponent becomes 1:1 (Figure 18). The shifts of the $\alpha_{2}$ peak temperature are summarized in Figure 19, where the peak temperature was not determined for PMDG-PMLG blend films with a $\mathrm{D}$ - and L-component ratio of $2: 1$ and $1: 1$ since the peak temperature is above $270^{\circ} \mathrm{C}$. The relaxation mechanism associated with the $\alpha_{2}$ loss peak must therefore be explained in consideration of the shift of peak temperature. Mechanical dispersion and loss peak occur at that temperature where molecular motion becomes activated at a frequency corresponding to that of the exciting mechanical force. Presently, there are no theoretical methods to predict this temperature for the molecular motions of $\alpha$-helices. However, if this $\alpha_{2}$ dispersion is due to the molecular motion of $\alpha$-helices, a significant shift of the peak temperature shown in Figure 19 should be reflected at the temperature where plots of the spacing of equatorial reflections vs. temperature exhibit a break attributable to the onset of molecular motion of $\alpha$-helices. In Figure 20, the spacing-temperature plots are shown for M-90 and PMDGPMLG racemic film. The observed temperature at the break is the same as that for the chloroform cast PMDG film. Thus, the $\alpha_{2}$ dispersion does not seem to be associated with any molecular motion of $\alpha$-helices in phase C.

In order to explain the shift of $\alpha_{2}$ peak temperature, another mechanism of relaxation for $\alpha_{2}$ dispersion may be considered. We found that, for the $m$-cresol cast films, the crystallite size increased

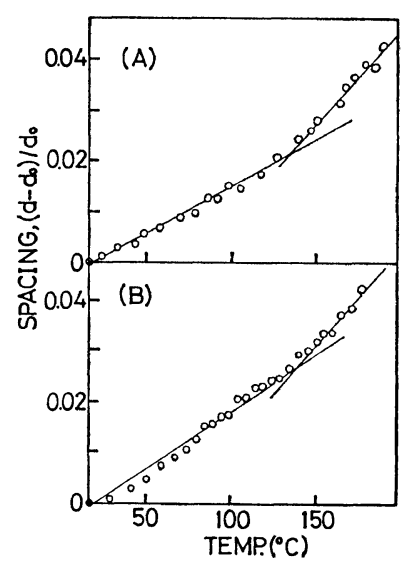

Figure 20. Temperature dependence of spacing of the (200) reflection for (A) PMDG films cast from $m$ cresol solution at $90^{\circ} \mathrm{C}$ and (B) PMDG-PMLG racemic film cast from chloroform solution. $d_{0}$ is the value of $d$ at $20^{\circ} \mathrm{C}$.

with an increase in the casting temperature, and assumed that the division of the crystallites in phase $C$ should occur as a result of the collapse of the cholesteric liquid crystal structure (Figure 8). On comparison of Figure 7 with Figure 19, it is obvious that a remarkable shift to a higher temperature of $\alpha_{2}$ peak is caused by the increasing crystallite size. On the other hand, it is well known that the pitch of cholesteric phase in PBDG -PBLG solutions increases as the ratio of $\mathrm{D}$ - and L-component becomes $1: 1 .^{29,30}$ This increase of the cholesteric pitch is due to the mixing of Dand L-molecules forming a cholesteric macrohelix in an opposite sense to each other, and was also observed for PMDG-PMLG chloroform solutions. Thus, for PMDG-PMLG blend films it is also likely that the crystallite size may increase in a similar process as is assumed for $m$-cresol cast films and the shift to the higher temperature of $\alpha_{2}$ peak may be caused by an increase in crystallite size, although crystallite size was not determined by the symmetrical-reflection methods because of the poor development of the equatorial reflections. As the relaxation mechanism in phase $\mathrm{C}$ consisting of a mosic structure, we may propose a mutual slip or mutual displacement of crystallites, caused by shearing of mosic crystallites. This sort of relaxation is not to be observed in phase I and phase M. The dependence of $\alpha_{2}$ loss peak temperature on 


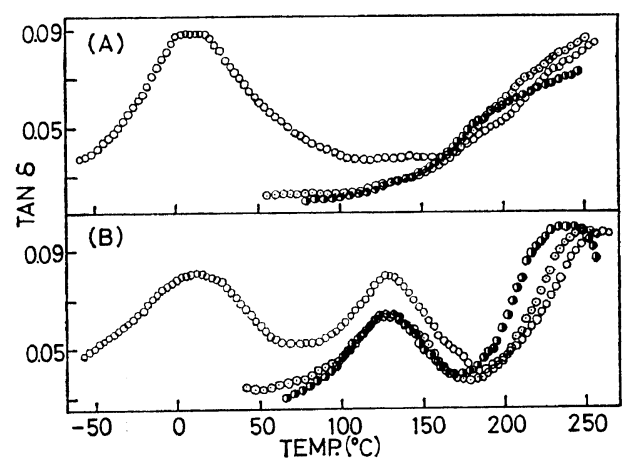

Figure 21. Plots of $\tan \delta v s$. temperature for the racemic films cast from (A) chloroform solution and (B) EDC solution. Data obtained at $110(\bigcirc), 11(\odot)$, and $3.5(\mathrm{O}) \mathrm{Hz}$.

crystallite size as established from Figures 7 and 19, seems likely. Further investigation, however, is necessary to clarify the relaxation mechanism for $\alpha_{1}$ dispersion.

The mechanical properties for PMDG-PMLG racemic film cast from EDC solution, are characterized by two loss peaks at about 130 and $240^{\circ} \mathrm{C}$ (see Figure 21). The racemic film cast from DMF solution also showed the same properties. These two peaks cannot reasonably be categorized into the loss peaks mentioned above. When the structure-property relationship of racemic films is considered, or when the interaction of D- and L-molecules is considered, it is of interest that the mechanical properties of racemic films cast from EDC or DMF solution are very different from those of films cast from chloroform solution.

Acknowledgment. This study was supported by a grant for scientific research (Kagaku-Kenkyuhi) from the Ministry of Education, Japan.

\section{REFFERENCES}

1. P. Doty, J. H. Bradbury, and A. M. Holtzer, $J$. Am. Chem. Soc., 78, 947 (1956).

2. A. Nakajima, T. Fujiwara, T. Hayashi, and K. Kaji, Biopolymers, 12, 2681 (1973).

3. J. Watanabe and I. Uematsu, Polym. J., 9, 195 (1977).
4. T. Kajiyama, K. Kuroishi, and M. Takayanagi, $J$. Macromol. Sci.-Phys., B 11, 121 (1975).

5. Y. Mohadger and G. Wilkes, J. Polym. Sci., 14, 963 (1976).

6. J. Watanabe, S. Sasaki, and I. Uematsu, Polym. J., 9, 451 (1977).

7. C. Robinson, Mol. Cryst., 1, 467 (1966).

8. T. Noda, Y. Abe, and R. Sakamoto, Kobunshi Ronbunshu, 31, 203 (1974).

9. J. Watanabe, S. Sasaki, and I. Uematsu, Polym. J., 9, 337 (1977).

10. J. Watanabe, K. Imai, and I. Uematsu, to be published.

11. R. S. Stein and M. B. Rhodes, J. Appl. Phys., 31, 1873 (1960).

12. Hl. de Vries, Acta Cryst., 4, 219 (1951).

13. T. L. Fergason, Mol. Cryst., 1, 293 (1966).

14. S. Ishikawa, T. Kurita, and E. Suzuki, J. Polym. Sci., Part A, 2, 2349 (1964).

15. G. Wilkes, Y. Mohadger, and W. Holaday, ibid., 14, 251 (1976).

16. S. Ishikawa, ibid., Part A, 3, 4075 (1965).

17. S. Sasaki, M. Hikata, and I. Uematsu, Rep. Prog. Polym. Phys. Jpn., 20, (1977).

18. A. C. Neville and S. Caveney, Biol. Rev., 44, 531 (1969).

19. Y. Bouligand, Tissue and Cell, 4, 189 (1972).

20. H. Hosemann and W. Wilke, Macromol. Chem., 118, 230 (1968).

21. L. E. Alexander, "X-ray Diffraction Methods in Polymer Science," John Wiley and Sons, Inc., New York, N. Y., 1969, Chapter 7.

22. N. Mori, Y. Yoshikawa, Y. Uematsu, and I. Uematsu, Rep. Prog. Polym. Phys. Jpn., 17, 625 (1974).

23. A. Miller and D. A. D. Parry, Polymer, 15, 706 (1974).

24. A. Elliott, R. D. B. Fraser, and T. P. MacRae, J. Mol. Biol., 11, 821 (1965).

25. Y. Yoshida, S. Sakurai, T. Okuda, and Y. Takagi, J. Am. Chem. Soc., 84, 3590 (1962).

26. J. Watanabe, S. Ooishi, S. Sasaki, and I. Uematsu, to be published.

27. E. S. Clark and L. T. Muus, Z. Kristallogr., 117, 108 (1962).

28. S. Sasaki, T. Kamata, and I. Uematsu, to be published.

29. N. Mori, Y. Uematsu, and I. Uematsu, Rep. Prog, Polym. Phys. Jpn., 16, 619 (1973).

30. C. Robinson and J. C. Ward, Nature, 180, 1183 (1957). 